\title{
An investigation of (nonadditive) scattering invariants in classical mechanics and quantum theory by differential topological methods
}

\author{
Manfred Requardt \\ Institut für Theoretische Physik der Universität Göttingen, Bunsenstrasse 9, 34 Göttingen, West Germany
}

(Received 20 March 1986; accepted for publication 8 April 1987)

\begin{abstract}
The structure of invariants of the scattering transformation in (relativistic and nonrelativistic) classical mechanics and quantum theory is investigated and a constructive approach to finding and classifying them by exploiting and developing certain differential topological methods is provided. While, in the form of various by-products, results about the perhaps better known so-called additive scattering invariants are (re)derived, the primary concern here is with the less well-known nonadditive (i.e., several particle) conserved quantities.
\end{abstract}

\section{INTRODUCTION}

The computation of the so-called integrals of motion in the classical mechanics of mass points is both a very old and (at least in general) difficult problem. It is well known that the number of independent integrals of motion for a closed mechanical system with $n$ degrees of freedom is $2 n-1$ (cf. Ref. 1) with an additive time constant being eliminated. For a Hamiltonian system a function $f \in C^{1}$ depending on the positions $\left\{q_{k}\right\}$ and momenta $\left\{p_{k}\right\}$ is an invariant along every path of motion iff

$$
\frac{d f}{d t}=\frac{\partial f}{\partial t}+\{H, f\}=0
$$

with $H$ the Hamiltonian and

$$
\{H, f\}=\sum_{k} \frac{\partial H}{\partial_{p_{k}}} \cdot \frac{\partial f}{\partial_{q_{k}}}-\frac{\partial F}{\partial_{q_{k}}} \cdot \frac{\partial f}{\partial_{p_{k}}} .
$$

Since we are in the following primarily dealing with systems in the usual Cartesian space we alter the notation slightly denoting positions by $x_{k}$, momenta by $p_{k}, x_{k}, p_{k} \in \mathbb{R}^{3}$ with $p_{k}=m_{k} \cdot \dot{x}_{k}$. This implies that we will not rely on the heavy abstract machinery being developed, for example, in the book of $\mathrm{Abraham}^{2}$ and more recent ones devoted to the study of the properties of general dynamical systems. Instead we would like to emphasize a certain conceptual relationship between the things we will discuss and related problems in quantum scattering theory and quantum field theory.

In this more general context one is interested in symmetries of the scattering matrix $S$ and how classical mechanics fits in a very natural way into this general scheme. One can then ask a slightly more general question. Instead of looking for physical quantities conserved along the whole path of the system (with the necessary modifications of this picture in quantum theory) one can direct one's attention to symmetries or invariants of the $S$ matrix, that is, observables asymptotically invariant under time evolution and approaching the same limits along each trajectory for time $t \rightarrow \pm \infty$. Investigations of the long time phenomena within classical mechanics by means of notions and strategies developed originally in quantum scattering theory can be found, e.g., in Refs. 3-6. We want, however, to remark that our approach is so general that it can be easily extended to more abstract dynamical systems.

We assume an interacting and a free time evolution to be given defining flows $\phi_{t}, \phi_{t}^{0}$ :

$$
\phi_{t}:(x(0), p(0)) \rightarrow(x(t), p(t)), \quad x, p \in \mathbb{R}^{3 n} .
$$

We define the so-called "Möller transformations" $\Omega_{ \pm}$,

$$
\Omega_{ \pm}:=\lim _{t \rightarrow \mp \infty} \phi_{-t} \cdot \phi_{t}^{0},
$$

assumed to exist on certain sets $D_{ \pm} \subset \mathbb{R}^{6 n}$, which we expect to be the set of initial conditions of all the free orbits up to certain sets of Lebesque measure zero.

As in quantum theory the situation can be relatively easily controlled in the two-body case or for single particle scattering in an external potential. For the $n$-particle case one has in principle to admit for the possibility of various channels, i.e., clusters of "bound" particles moving freely in the limits $t \rightarrow \pm \infty$. While in our approach the number of particles (resp. clusters) moving to infinity for $t \rightarrow+\infty$ is allowed to differ from the number of "ingoing" ones, we prefer to not overburden the formalism with these details and assume that all particles are unbound as $t \rightarrow \pm \infty$. (The necessary machinery to deal with bound clusters of particles, e.g., channel Hamiltonians, cluster decompositions and all that, can be found in Ref. 5 and is basically the same as in quantum scattering.) Thus we have to exclude the initial conditions for the free comparison dynamics (note that these are the phase space coordinates at $t=0$ ), where some of the relative momenta are zero. With this proviso we assume the admissible initial conditions to be $\mathbb{R}^{6 n}$ up to sets of measure zero (as to more details and the various notions of asymptotic completeness in the single particle case we refer the reader, for example, to Chap. XI.2 of Ref. 4).

The $S$ transformation defined by

$$
S:=\left(\Omega_{-}\right)^{(-1)} \cdot \Omega_{+}
$$

then maps free asymptotes at $t=-\infty$ onto the corresponding ones at $t=+\infty$. The free orbits

$$
x_{i}^{0}(t)=x_{i}^{o}+p_{i} / m_{i} \cdot t
$$

are mapped by the Möller transformations onto the corresponding time zero coordinates of the interacting system

$$
\Omega_{ \pm}:\left\{x_{i}^{0}, p_{i}\right\} \rightarrow\left\{x_{i}(0), p_{i}(0)\right\}
$$

called

$$
\left\{x_{i}^{\text {in }}, p_{i}^{\text {in }}\right\} \quad\left(\text { resp. }\left\{x_{i}^{\text {out }}, p_{i}^{\text {out }}\right\}\right)
$$

and the $S$ transformation 


\section{$S:\left\{x_{1} p_{1}, \ldots, x_{n} p_{n}\right\} \rightarrow\left\{x_{1}^{\prime} p_{1}^{\prime}, \ldots, x_{n}^{\prime} p_{n}^{\prime}\right\}$}

(where for notational simplicity we supress the superscript $0)$.

Remark: We discuss only the case of short-range potentials. For long-range potentials the asymptotes have to be slightly modified (cf. Ref. 4 Chap. XI.9).

Definition 1: Invariants of the $S$ transformation are functions $F$ such that (s.t.)

(i) $F\left(x_{1} p_{1}, \ldots, x_{n} p_{n}\right)=F\left(x_{1}^{\prime} p_{1}^{\prime}, \ldots, x_{n}^{\prime} p_{n}^{\prime}\right)$,

where the coordinates on the lhs and rhs are the pairs occurring in a scattering event with the additional property of being constant along free paths; i.e.,

(ii) $F\left(x_{1}^{o}(t) p_{1}, \ldots, x_{n}^{0}(t) p_{n}\right)=F\left(x_{1}^{o}\left(t^{\prime}\right) p_{1}, \ldots, x_{n}^{o}\left(t^{\prime}\right) p_{n}\right)$

(in principle we could admit an $F^{\prime}, n^{\prime}$ different from $F, n$ on the rhs).

Perhaps a little bit surprisingly, quantum and quantum field theory (without spin) can be treated along the same lines. We assume a self-adjoint operator $Q$ to be given on the $n$-particle states of the free time evolution. If $Q$ is the generator of a symmetry it usually can be represented on the free states by a certain sum of terms consisting of products of functions $f_{i}\left(x_{1}, \ldots, x_{n}\right)$ and expressions in $\partial_{x_{1}}, \ldots, \partial_{x_{n}}$, i.e.,

$$
Q \hat{=} \sum_{i} f_{i}\left(x_{1}, \ldots, x_{n}\right) \cdot P_{i}\left(\partial_{1}, \ldots, \partial_{n}\right) .
$$

If $Q$ commutes with $S$ we have

$$
(\varphi, S \cdot Q \psi)=(Q \varphi, S \psi),
$$

which implies for the scattering amplitude $S\left(x_{1}, \ldots, x_{n}\right.$; $\left.x_{1}^{\prime}, \ldots, x_{n}^{\prime}\right)$ (actually a certain distribution)

$$
\begin{gathered}
\left\{\sum_{i} f_{i}\left(x_{1}, \ldots, x_{n}\right), P_{i}\left(\partial_{x_{1}}, \ldots, \partial_{x_{n}}\right)-\sum_{i} f_{i}\left(x_{1}^{\prime}, \ldots, x_{n}^{\prime}\right)\right. \\
\left.\quad \times P_{i}\left(\partial_{x_{i}}, \ldots, \partial_{x_{n}}\right)\right\} \cdot S\left(x_{1}, \ldots, x_{n} ; x_{1}^{\prime}, \ldots, x_{n}^{\prime}\right) \equiv 0
\end{gathered}
$$

and a corresponding expression in momentum space which is particularly useful for symmetries commuting with the space translations. In this special case we get

$$
\left\{P\left(p_{1}, \ldots, p_{n}\right)-P\left(p_{1}^{\prime}, \ldots, p_{n}^{\prime}\right)\right\} \cdot S\left(p ; p^{\prime}\right) \equiv 0 .
$$

If there is scattering at all, i.e., a certain open set on the scattering manifold where $S\left(p ; p^{\prime}\right)$ is nontrivial, the expression in the curly brackets has to vanish identically on this set, a situation completely analogous to the classical one.

The investigation of symmetries of the $S$ matrix focussed mainly on so-called additive or summation invariants, notions being explained later, leading to perhaps a little bit puzzling: "no go theorems," usually of the tenor that there exist no more additive invariants (apart from inner symmetries) than the a priori ones already known. In quantum field theory many papers were initiated by a result of this type by Coleman and Mandula ${ }^{7}$ (cf. also Refs. 8 and 9 and previously Ref. 10). As to classical mechanics the history is of course much longer and we want to give only a few references. Results of this type have been of particular interest in kinetic gas theory (cf., e.g., Refs. 11 and 12), and belonged in a certain sense to the general folklore in this field. The situation was then more carefully studied by Grad, ${ }^{13}$ who remarks that the situation is far from obvious, and quite recently by Amigo and Reeh, ${ }^{14}$ where further references (e.g., for relativistic dynamics) can be found. In this context, with particular emphasis on relativistic particle scattering, one should also mention the original approach to the whole subject matter given in Ref. 15.

While our investigation will, in the form of various byproducts, also yield results about additive conservation laws its main impetus is, however, directed towards the much less well known but probably more important regime of nonadditive scattering invariants. Since, for example, all $2 n-1$ initial conditions are by definition invariants of motion in classical mechanics [when being expressed as functions of the actual $\left.x_{i}(t), p_{i}(t)\right]$, while, on the other side, the number of additive ones usually does not exceed 10 , it is quite apparent that the former set is not empty. It is, however, both difficult to extract them from the concretely given model theories and, a fortiori, to characterize them by means of more general principles. Some steps in this direction can, e.g., be found in the classical book of Whittaker, ${ }^{16}$ the perhaps most notable result in this direction being the theorem of Bruns, viz., there are no other algebraic invariants of motion in celestial mechanics of three bodies than the already known ones. [Note that (i) in the case of two bodies there does exist an additional one, namely the so-called RungeLenz vector; and (ii) the emphasis lies on "algebraic," which implies that while additional invariants do exist they are necessarily of a complicated (transcendental) type and therefore difficult to find.]

In quantum field theory the situation is (understandably) less transparent, in particular concerning existence and properties of objects corresponding to the nonadditive scattering invariants of classical mechanics. A possibly analogous role might be played by multilocal conserved quantities (called charges in this field). These objects have been found in some lower-dimensional models and in a recent paper Buchholz, Lopuszanski, and Rabsztyn ${ }^{17}$ (see also Refs. 18 and 19) try to develop an approach allowing us to systematically study them in the physical relevant dimension 4. It may be promising to try to relate these ideas with our strategy, which is different, in the future.

Our approach, in contrast to most of the papers mentioned above, carries a distinctive differential topological flavor. Its advantages are (in our view) that it allows us to study these and more general questions on a considerably broader scale and in a concise and unified manner, i.e., the same methods work in classical mechanics, quantum theory, and quantum field theory (QFT). Even in the much better understood situation of additive invariants it sheds some new light upon some of the perhaps more hidden aspects of the problems under discussion.

To mention a few: (i) it does not need the existence of a regime where particles scatter only elastically; (ii) the somewhat hidden but (in fact) for many conclusions crucial and nontrivial assumption of the existence of certain suitable open sets on the scattering manifold being coverable by real scattering events is brought to light, leading, on the other side, to a fine structure within the class of scattering invar- 
iants; and (iii) it might perhaps be easier to extend our approach to more complicated invariants in QFT having, e.g., a tensor (resp. spinor) character of a higher degree. This represents still a major obstacle in extending the classical Coleman-Mandula result to higher charges. Also (iv) since neither the particle number nor the shape of the function $F$ has to be the same for in- (resp. out-) states, the approach can be easily extended to objects (charges) which do not commute with the $S$ matrix! This topic and the extension to general charges in quantum field theory will, however, be given elsewhere.

The paper is organized as follows: In Sec. II we transform the problem into a purely mathematical one thus displaying what sort of treatment is actually the appropriate one, what belongs to the physical input, and what is the mathematical machinery. We then discuss in Sec. III in a first step invariants depending only on the momenta of the scattering partners and derive various structure theorems for them as, e.g., every nonadditive invariant depending only on the momenta is a function of overall energy and momentum (in most cases even a polynomial). In Sec. IV we carefully analyze the physical side of the problem, in particular, the structure of the set of in- and out-states being connectable by the scattering transformation. Section $V$ deals with the structure of invariants depending on the momenta, positions, and the time. The results are, however, less complete and cover only the field of classical mechanics (in order not to overburden the paper). It is exactly at this point where future work has to set in. In the last section, which has more the character of an appendix, we discuss an explicit counterexample against the usual physical intuition, i.e., the potential $V(r) \sim r^{-2}$.

\section{THE MATHEMATICAL SIDE OF THE PROBLEM}

We begin with the subclass of invariants depending only on the momenta of the asymptotic particles. Furthermore, we restrict ourselves, for the time being, to functions from $C^{1}\left(\mathbb{R}^{3 n}\right)$. It is not clear to us whether one really loses something by not considering more nasty functions in this special context. In any case, by smearing with appropriate test functions, one can usually extend the results to more general invariants (if there are any) (see, e.g., Ref. 14).

This question is, however, not purely academic. Take, e.g., one of the Cauchy equations over $\mathbb{R}$ :

$$
f(x+y)=f(x)+f(y) .
$$

Assuming $f$ to be in $C^{1}$ the construction of a solution is very easy. We have

$$
f^{\prime}(x+y)=f^{\prime}(x)=f^{\prime}(y), \quad f(0)=f(0)+f(0),
$$

from which

$$
f(0)=0, f^{\prime}(x)=\text { const, i.e., } f(x)=a \cdot x,
$$

follows. In a next step one usually tries to prove that possible solutions lying in a more general class are automatically differentiable. There is, for example, the result that a solution being locally Lebesgue integrable is already $\in C^{1}$. On the other hand there do exist nonmeasurable solutions(!) (cf. the history of this equation given in Ref. 20, Chap. 14.2 or in
Ref. 15).

Let $p_{i}, \epsilon_{i}\left(p_{i}\right), i=1, \ldots, n$ be the momenta and energies of the asymptotic particles. Energy-momentum conservation then forces the ingoing and outgoing momenta to lie in a certain subset of $\mathbb{R}^{3 n} \times \mathbb{R}^{3 n}$, i.e., we present the following definition.

Definition 2: The scattering manifold $M \subset \mathbb{R}^{3 n} \times \mathbb{R}^{3 n}$ in momentum space is defined by the four constraints

$$
\begin{aligned}
& \sum_{i=1}^{n} p_{i}-\sum_{i=1}^{n} p_{i}^{\prime}=0, \\
& \sum_{i=1}^{n} \epsilon_{i}\left(p_{i}\right)-\sum_{i=1}^{n} \epsilon_{i}\left(p_{i}^{\prime}\right)=0,
\end{aligned}
$$

with $p_{i} \in \mathbb{R}^{3}, \epsilon_{i}\left(p_{i}\right) \in C^{1}$, away from possibly a certain set of discrete points. The corresponding Jacobi matrix is assumed to have rank 4 , i.e., the constraints are assumed to be independent. In more modern language,

$$
\begin{aligned}
f: & \mathbb{R}^{6 n} \rightarrow \mathbb{R}^{4}, \\
& \left(p_{1}, \ldots, p_{n} ; p_{1}^{\prime}, \ldots, p_{n}^{\prime}\right) \\
& \rightarrow\left(\sum p_{i}-\sum p_{i}^{\prime}, \sum \epsilon_{i}\left(p_{i}\right)-\sum\left(p_{i}^{\prime}\right)\right)
\end{aligned}
$$

does not have $0 \in \mathbb{R}^{4}$ as a critical value. (As to this notion cf., e.g., Ref. 20.)

Remarks: (i) In applications $\epsilon(p)$ is usually $\in C^{\infty}$ away from possibly $p=0$, e.g., $\epsilon(p)=c \cdot p^{2}, \sqrt{p^{2}+m^{2}}$, etc., $\epsilon(p)=c \cdot|p|$ may serve as an example for an $\epsilon(p)$ not $\epsilon C^{1}$ in $p=0$.

(ii) As to the assumed independence of the above four constraints, one should say that this is the usual state of affairs (e.g., for particles with nonzero mass). There exist, however, even in physics, illuminating counterexamples. In principle two situations can occur in general:

( $\alpha$ ) rank $f<4$ at a point but is, nevertheless, locally constant (the rank is locally lower continuous anyway);

( $\beta$ ) rank $f<4$ but is locally nonconstant, i.e., it increases (discontinuously) in every neighborhood of that point.

In case $(\alpha)$ the situation is still relatively smooth. The codimension of $M$ is simply $<4$. However, $(\beta)$ is much more singular. The codimension of $M$ (which, in general, may even no longer be a true submanifold of $\mathbb{R}^{m \cdot n}$ ) may increase abruptly to values $>4$ at some points s.t. the set $M$ may turn out to be much smaller than expected. This can also happen in physics: Take, e.g., disintegration of a zero mass particle into two others:

$p_{1}=p_{1}^{\prime}+p_{2}^{\prime}, \quad\left|p_{1}\right|=\left|p_{1}^{\prime}\right|+\left|p_{2}^{\prime}\right|$ implying $p_{1}^{\prime} \| p_{2}^{\prime}$.

That is, we get actually five constraints for the momenta $p_{1}$, $p_{1}^{\prime}, p_{2}^{\prime}$ from the four equations (2.4). The codimension of $M$ is now 5 instead of 4 (one of the typical phase space constraints in photon scattering). Stated differently, the map (2.5) has 0 as a critical value and $(\beta)$ applies. We assume this phenomenon to be absent in the following.

As was already remarked in Ref. 14 the whole manifold $M$ is not usually accessible to concrete scattering experiments. On the other hand this is an important point since 
almost all the classical proofs relied heavily on this assumption. Solutions of the equations of motion are usually given uniquely by their initial conditions. But one has a certain freedom in varying, e.g., the impact parameter while keeping the ingoing momenta fixed, thereby varying the outgoing momenta. These physical aspects of the problem will be discussed in detail in Sec. IV. So let us for the moment simply assume that, while the whole $M$ usually cannot be covered by scattering events for a given fixed interaction between the particles, there exists at least an open set $U \subset M$ corresponding to a concrete scattering situation. Thus we have the following definition.

Definition 3: A function $F\left(p_{1}, \ldots, p_{n}\right) \in C^{1}\left(\mathbf{R}^{3 n}\right)$ is called an invariant of the scattering transformation of the first kind iff for a certain open subset $U \subset M$ we have the relation

$$
F(p)-F\left(p^{\prime}\right) \equiv 0 \text { for all }\left(p, p^{\prime}\right) \in U
$$

[with $p, p^{\prime}$ standing for the $n$-tuples $\left.\left(p_{1}, \ldots, p_{n}\right), p_{1}^{\prime}, \ldots, p_{n}^{\prime}\right)$ ] . This implies $\operatorname{dim}(U)=\operatorname{dim}(M)=(6 n-4)$. The general mathematical situation is, however, more involved. With $P^{\prime}:=\Sigma p_{i}^{(\prime)}, E^{(\prime)}:=\Sigma \epsilon_{i}\left(p_{i}^{(\prime)}\right), M$ was defined as the intersection of the four hypersurfaces given by energy-momentum conservation. If one looks for further conserved quantities, say $F$, what has to be usually expected is the relation $F(p)=F\left(p^{\prime}\right)$ to hold on $M$ only on a submanifold of dimension $<6 n-4-1$, the intersection of the hypersurface given by the additional conservation law with $M$. That is, we have the following.

Definition 4: The function $F$, given in Definition 3, is called an invariant of the second kind iff relation $F(p) \equiv F\left(p^{\prime}\right)$ holds only a subset $U^{\prime} \subset U \subset M$ of dimension $d<6 \cdot n-5$.

Geometric observation: If the situation is the one described in Definition $3 M$ is at least locally contained in the hypersurface defined by $F$. Definition 4 describes the phenomenon that this hypersurface hits $M$ transversally, thus reducing the dimension by at least 1 (as to more details concerning these notions cf. Ref. 21.

Physical observation: Let $M_{p} \subset M$ denote the (physical) submanifold which can be covered by real scattering experiments. Under the assumption of Definition $3 \operatorname{dim}(M)_{p}$ $=\operatorname{dim}(M)$. Since the momenta of the ingoing particles can always be freely chosen we have $\operatorname{dim}(M)_{p} \geqslant 3 n$ in general. We show in Sec. IV that in the pure momentum case $\operatorname{dim}(M)_{p}=\operatorname{dim}(M)$. In the case of more general invariants this will, however, never hold! In that case, i.e., $\operatorname{dim}(M)_{p}<\operatorname{dim}(M)$, Definition 4 becomes relevant.

Whether there are such invariants of the second kind requires a careful analysis of the physical scattering situation and this will be given in Sec. IV. In any case there exists a very useful structure theorem that allows us to discriminate between these two possibilities. For its proof we need the following simple lemma.

Lemma 1: Let $f \in C^{1}$ be a function of an open neighborhood $\widetilde{U}$ of $0 \in \mathbb{R}^{n} \times \mathbb{R}^{k}$, the coordinates denoted by $\left(x_{1}, \ldots, x_{n}\right.$; $\left.y_{1}, \ldots, y_{k}\right)$. The following is assumed to hold:

$$
f \mid \widetilde{U} \cap\left(\mathbb{R}^{n} \times\{0\}\right) \equiv 0 .
$$

Then $f$ can be written in a full open neighborhood $\widetilde{U}^{\prime} \subset \widetilde{U}$ of $0 \in \mathbf{R}^{n} \times \mathbf{R}^{k}$ in the form $f=\sum_{i=1}^{k} y_{i} \cdot f_{i}, \quad f_{i} \in C$ with respect to $y$.

\section{Proof:}

$$
\begin{aligned}
f(x, y) & =f(x, 0)+\int_{0}^{1} \frac{d}{d t} f(x, t \cdot y) d t \\
& =\sum_{i=1}^{k} y_{i} \cdot \int_{0}^{1} \partial_{t y_{i}} f(x, t \cdot y) d t .
\end{aligned}
$$

Let now $U$ be open on $M$ s.t. $F(p)=F\left(p^{\prime}\right)$ holds on $U$. Since $M$ is given by the relations

$$
P-P^{\prime} \equiv 0, \quad E-E^{\prime} \equiv 0,
$$

with the corresponding Jacobi matrix having rank 4 on $U$, we can choose local coordinates in an open neighborhood $\widetilde{U} \supset U, \widetilde{U}$ open in $\mathbf{R}^{3 n} \times \mathbf{R}^{3 n}$ ! We obtain

$$
\left(\tilde{x}_{1}, \ldots, \tilde{x}_{6 n-4} ; \tilde{y}_{1}, \ldots, \tilde{y}_{4}\right)
$$

s.t.

$$
\begin{aligned}
& \tilde{y}_{1}:=P_{1}-P_{1}^{\prime}, \ldots, \\
& \tilde{y}_{3}:=P_{3}-P_{3}^{\prime}, \quad \tilde{y}_{4}:=E(p)-E\left(p^{\prime}\right),
\end{aligned}
$$

that is, with $\tilde{y}_{l}$ a basis of local coordinates being normal to $M$. With the help of Lemma 1 we can now prove the following proposition.

Proposition 1: Let $U$ be open on $M$ s.t. $F(p)=F\left(p^{\prime}\right)$ on $U$ holds with $F$ given in Definition 3. We have then in a neighborhood $\widetilde{U} \supset U, \widetilde{U}$ open in $\mathbf{R}^{3 n} \times \mathbf{R}^{3 n}$, the representation

$$
\begin{aligned}
F(p)-F\left(p^{\prime}\right)= & \sum_{i=1}^{3}\left(P_{i}-P_{i}^{\prime}\right) \cdot G_{i}\left(p, p^{\prime}\right) \\
& +\left(E(p)-E\left(p^{\prime}\right)\right) \cdot G_{4}\left(p, p^{\prime}\right)
\end{aligned}
$$

with $G_{i}, G_{4} \in C\left(\mathbf{R}^{3 n} \times \mathbf{R}^{3 n}\right), P, P^{\prime}, E(p), E\left(p^{\prime}\right)$ the overall momentum and energy of the ingoing and outgoing particles, and $P_{i}$, e.g., denoting the $i$ th component of $P$.

Proof: $\widetilde{U}$ is diffeomorphic to a neighborhood of $0 \in \mathbb{R}^{6 n-4} \times \mathbf{R}^{4}$ with $\tilde{y}_{i}=P_{i}-P_{i}^{\prime}, \tilde{y}_{4}:=E-E^{\prime}$ locally spanning $\mathbf{R}^{4}$. Here $F(p)-F\left(p^{\prime}\right)$ corresponds to a certain function $\widetilde{F}(\tilde{x}, \tilde{y})$ defined around $0 \in \mathbf{R}^{6 n-4} \times \mathbf{R}^{4}$ with $\widetilde{F} \mid\left(\widetilde{U} \subset \mathbb{R}^{6 n-4} \times\{0\}\right) \equiv 0$ (since $F$ is supposed to be conserved on $M$ ). By Lemma 1 we see that $F$ has a representation

$$
\widetilde{F}(\tilde{x}, \tilde{y})=\sum \tilde{y}_{i} \cdot G(\tilde{x}, \tilde{y})+\tilde{y}_{4} \cdot G_{4}(\tilde{x}, \tilde{y}) .
$$

Reinserting the original coordinates $\left\{p_{1}, \ldots, p_{n} ; p_{1}^{\prime}, \ldots, p_{n}^{\prime}\right\}$ proves the statement.

If we have the identity $F(p)-F\left(p^{\prime}\right)$ only on a submanifold of smaller dimension than $M$ itself the above local coordinate system would be not exhaustive. The local coordinate which is missing may just belong to a hidden independent conservation law or to $F$ itself. In any case, $F(p)-F\left(p^{\prime}\right)$ cannot be represented in the form (2.12) when this happens to be the case.

\section{A CONSTRUCTIVE DETERMINATION OF THE FUNCTIONAL FORM OF (NON)ADDITIVE INVARIANTS (PURE MOMENTUM CASE)}

This section contains the bulk of the technical aspects of our approach together with a complete classification of gen- 
eral invariants depending only on the momenta. Invariants depending also on the positions are treated in Sec. V. A crucial role will be played by our structure theorem (2.12). We will exploit the peculiar form of (2.12), i.e., that the lhs is the difference of two functions depending solely on either $p$ or $p^{\prime}$. To that end we will differentiate both sides with respect to $p_{i}, p_{j}^{\prime}, i, j=1, \ldots, n$, assuming, in order that the $G_{i}$ 's be $\in C^{1}$, that $F \in C^{2}$. We then get

$$
\begin{aligned}
\partial_{p_{i}} F(p)= & \mathbf{G}\left(p, p^{\prime}\right)+\left(P_{v}-P_{v}^{\prime}\right) \cdot \partial_{p_{i}} G_{v}\left(p, p^{\prime}\right) \\
& +\partial_{p_{i}} \epsilon_{i}\left(p_{i}\right) \cdot G_{4}\left(p, p^{\prime}\right) \\
& +\left(E(p)-E\left(p^{\prime}\right)\right) \cdot \partial_{p_{i}} G_{4}\left(p, p^{\prime}\right),
\end{aligned}
$$

with $\mathbf{G}$ the vector with components $G_{\nu}, v=1,2,3$, and an analogous result for $p_{j}^{\prime}$.

Choosing $\left(p, p^{\prime}\right)$ to lie a fortiori on $M$ we have $\left(P_{v}-P_{v}^{\prime}\right)=\left(E(p)-E\left(p^{\prime}\right)\right)=0$, that is

$$
\begin{aligned}
& \partial_{p_{i}} F(p) \mid U=\mathbf{G}\left(p, p^{\prime}\right)+\partial_{p_{i}} \epsilon_{i}\left(p_{i}\right) \cdot G_{4}\left(p, p^{\prime}\right), \\
& \partial_{p_{j}^{\prime}} F\left(p^{\prime}\right) \mid U=\mathbf{G}\left(p, p^{\prime}\right)+\partial_{p_{j}^{\prime}} \epsilon_{j}\left(p_{j}^{\prime}\right) \cdot G_{4}\left(p, p^{\prime}\right) .
\end{aligned}
$$

The expressions (3.2) and the corresponding ones in the more complicated situations dealt with in Sec. $V$ will turn out to be of particular use in calculating invariants. What we have in fact achieved by using this simple trick is that the lhs of (3.2) depends either solely on $p$ or $p^{\prime}$ while on the rhs there are terms, $\mathbf{G}, G_{4}$, which are independent of the subscript $i, j$ !

We proceed now as follows: In a first step we get

$$
\begin{aligned}
& \mathbf{G}\left(p, p^{\prime}\right)=-\partial_{p_{i}} \epsilon_{i}\left(p_{i}\right) \cdot G_{4}\left(p, p^{\prime}\right)+\partial_{p_{i}} F(p), \\
& \mathbf{G}\left(p, p^{\prime}\right)=-\partial_{p_{j}^{\prime}} \epsilon_{j}\left(p_{j}^{\prime}\right) \cdot G_{4}\left(p, p^{\prime}\right)+\partial_{p_{j}^{\prime}} F\left(p^{\prime}\right)
\end{aligned}
$$

which holds on $U \subset M$ and for all $i, j$. This expression can be exploited in various ways. The lhs is completely independent of the index $i$ (resp. $j$ ). So, taking the derivative in the first (second) expression with respect to $p_{i}, p_{j}\left(p_{l}^{\prime}, p_{k}^{\prime}\right)$, subtracting the corresponding equations from each other and bringing $G_{4}$ to the left-hand side we arrive at

$$
\begin{aligned}
G_{4}\left(p, p^{\prime}\right)= & \left(\partial_{p_{i}}^{(v)} F(p)-\partial_{p_{j}}^{(v)} F(p)\right) \\
& \times\left(\partial_{p_{i}}^{(v)} \epsilon_{i}\left(p_{i}\right)-\partial_{p_{j}}^{(v)} \epsilon_{j}\left(p_{j}\right)\right)^{-1}, \\
G_{4}\left(p, p^{\prime}\right)= & \left(\partial_{p_{i}^{(v)}}^{(v)} F\left(p^{\prime}\right)-\partial_{p_{k}^{\prime}}^{(v)} F\left(p^{\prime}\right)\right) \\
& \times\left(\partial_{p_{i}^{\prime}}^{(v)} \epsilon_{l}\left(p^{\prime}\right)-\partial_{p_{k}^{\prime}}^{(2)} \epsilon_{k}\left(p_{k}^{\prime}\right)\right)^{-1}
\end{aligned}
$$

(no summation over $\nu$ !), which holds on $U$ where the superscript $v$ denotes the $v$ th component of the gradient. Reinserting this into (3.3) we get a corresponding explicit expression for $\mathbf{G}$. We observe the remarkable fact that both the rhs of (3.4) and after having inserted the lhs of (3.4) into (3.3) the rhs of (3.3) depend either solely on $p$ or $p^{\prime}$ while $\mathbf{G}\left(p, p^{\prime}\right)$, $G_{4}\left(p, p^{\prime}\right)$ are, in principle, functions of both $p$ and $p^{\prime}$. This will be exploited in the following way.

While away from the scattering manifold, $M, \mathbf{G}, G_{4}$ are expected to depend both on $p$ and $p^{\prime}$, we see that on $M$, i.e., the subset of pairs $\left(p, p^{\prime}\right)$ which can be related via a scattering process, we have

$$
\begin{aligned}
& G_{v}\left(p, p^{\prime}\right)=G_{v}(p)=G_{v}\left(p^{\prime}\right), \\
& G_{4}\left(p, p^{\prime}\right)=G_{4}(p)=G_{4}\left(p^{\prime}\right) .
\end{aligned}
$$

But this is exactly the condition a scattering invariant has to fulfill! That is, we have the following theorem.

Theorem 1: With $F \in C^{2}$ being a scattering invariant on an open neighborhood $U$ on $M$, i.e., $F(p)=F\left(p^{\prime}\right),\left(p, p^{\prime}\right) \in U$, there exists a neighborhood $\widetilde{U} \supset U$, open in $\mathbb{R}^{3 n} \times \mathbb{R}^{3 n}$, s.t.

$F(p)-F\left(p^{\prime}\right)=G^{v} \cdot\left(P_{v}-P_{v}^{\prime}\right)+G^{4} \cdot\left(E(p)-E\left(p^{\prime}\right)\right)$

holds on $\widetilde{U}, G^{v}, G^{4} \in C^{1}$ with respect to $\left(p, p^{\prime}\right)$. Here $G^{v}$ and $G^{4}$ have the remarkable property that they are themselves scattering invariants, i.e., we have

$$
\left.\begin{array}{l}
G^{v}\left(p, p^{\prime}\right)=G^{v}(p)=G^{v}\left(p^{\prime}\right) \\
G^{4}\left(p, p^{\prime}\right)=G^{4}(p)=G^{4}\left(p^{\prime}\right)
\end{array}\right\}\left(p, p^{\prime}\right) \in \mathbf{M}
$$

Here $G^{v}$ and $G^{4}$ are explicitly given on $M$ by the expressions (3.3) and (3.4).

Before we proceed to deal with the more complex situation we would like to discuss the special class of so-called additive invariants.

Definition 5: An invariant, $F(p)$, of the scattering transformation is called additive iff

$$
F(p)=\sum_{i=1}^{n} f_{i}\left(p_{i}\right) \text {. }
$$

In this particular case the relations (3.3) and (3.4) can be readily exploited showing that $G^{v}$ and $G^{4}$ are on $M$ functions of any of the couples $\left(p_{i}, p_{j}\right)\left[\right.$ resp. $\left.\left(p_{l}^{\prime}, p_{k}^{\prime}\right)\right], i \neq j, k \neq l$. This shows that, in fact $G^{v}$ and $G_{4}$ cannot really depend on any of these couples on $M$, that is, we have

$$
G_{4}=\text { const, } G^{\nu}=\text { const on } M \text {. }
$$

By (3.2) we get

$$
\partial_{p_{i}}^{(v)} f_{i}\left(p_{i}\right)=C_{v}+\partial_{p_{i}} \epsilon_{i}\left(p_{i}\right) \cdot C_{4} \text { on } M \text {, }
$$

which implies

$$
f_{i}\left(p_{i}\right)=C_{v} \cdot p_{i}^{(v)}+C_{4} \epsilon_{i}\left(p_{i}\right)+C_{0, i},
$$

with $C_{v}, C_{4}$ being independent of the index $\{i\}$. That is, we have the following theorem.

Theorem 2: For an additive conserved quantity, $F(p)$ $=\Sigma_{i=1}^{n} f_{i}\left(p_{i}\right)$, on $U \subset M$ we have the relation (3.9), that is,

$$
F(p)=C_{v} \cdot P^{(v)}+C_{4} \cdot E(p)+C_{0},
$$

i.e., a superposition of momentum, energy, and a constant. We want to come now to the main topic of this investigation, i.e., the structure of nonadditive scattering invariants. This is an extremely difficult subject matter and very little is known. We mention in this context, e.g., the classical book of Whittaker, ${ }^{16}$ where, properly speaking, invariants of motion are discussed and Ref. 17 in the case of QFT. The machinery being developed in this chapter will turn out to be sophisticated enough to give an exhaustive answer in the restricted case of invariants depending only on the momenta. Many of the calculational steps can, however, be carried over to invariants depending also on the positions of the particles and the time but this will be a much more ambitious program with partial answers being given in Sec. V.

We have the following theorem.

Theorem 3: (i) Under the same assumptions as in Theorem 1 every scattering invariant depending only on the momenta is a function of overall energy $E$ and momentum $P$ provided that the map $\left\{p \rightarrow \partial_{p} \epsilon(p)\right\}$ is a homeomorphism 
a.e., i.e.,

$$
F\left(p_{1}, \ldots, p_{n}\right)=F\left(\sum \epsilon_{i}\left(p_{i}\right), \sum p_{i}\right)
$$

(ii) Furthermore, if $F$ is an $m$-particle observable with $f \in C^{m}$ and $m<n$, i.e.,

$$
F\left(p_{1}, \ldots, p_{n}\right)=\sum_{\left\{i_{1}, \ldots, i_{m}\right\}} f_{\cdots,\}}\left(p_{i_{1}}, \ldots, p_{i_{m}}\right),
$$

then $f$ is at most a polynomial of $m$ th order in the variables $p_{i}, \epsilon_{i}$ of the form (3.25).

Remarks: (i) Note that the result holds also in quantum field theory for translationally covariant charges.

(ii) The crucial part is (i), i.e., (3.11). The idea that also (ii) should hold (which is obvious for polynomials) was inspired by an observation made in Ref. 15 in the special case of relativistic three-particle scattering and a two-particle scattering invariant. The assumption $m<n$ is crucial for the proof. This is, however, not a series drawback since in many cases one can simply add more particles in order to make $n$ bigger than $m$ for fixed given $f\left(p_{1}, \ldots, p_{m}\right)$.

Proof: (i) As before we choose a special local coordinate system in $R^{3 n}$, the first four local coordinates being $P$ and $E$, the remaining $3 n-4$ being denoted by $\zeta:=\left\{\xi_{1}, \ldots, \zeta_{3 n-4}\right\}$ and consider $F$ as a function of $E, P, \xi$. Employing Eq. (3.2) we get $\left(\partial_{\xi} F:=\left(\partial_{\xi_{1}} F, \ldots, \partial_{\xi_{3 n-4}} F\right), \partial_{p_{i}} \xi:=\left(\partial_{p_{l}} \xi_{1}, \ldots\right)\right)$

$$
\begin{aligned}
& \partial_{P} F+\partial_{E} F \cdot \partial_{p_{i}} \epsilon\left(p_{i}\right)+\partial_{\zeta} F \cdot \partial_{p_{i}} \xi \\
& =\mathbf{G}+G_{4} \cdot \partial_{p_{i}} \epsilon\left(p_{i}\right) \text { for all } i, \\
& \begin{aligned}
\partial_{E} F \cdot\left(\partial_{p_{i}} \epsilon\left(p_{i}\right)-\partial_{p_{j}} \epsilon\left(p_{j}\right)\right)+\partial_{\zeta} F \cdot\left(\partial_{p_{i}} \xi-\partial_{p_{j}} \xi\right) \\
=G_{4} \cdot\left(\partial_{p_{i}} \epsilon\left(p_{i}\right)-\partial_{p_{j}} \epsilon\left(p_{j}\right)\right),
\end{aligned}
\end{aligned}
$$

implying

$$
\begin{aligned}
G_{4}= & \partial_{E} F+\partial_{\xi} F \cdot\left\{\left(\partial_{p_{i}^{\prime}} \xi-\partial_{p_{j}^{\prime}} \xi\right)\right. \\
& \left.\times\left(\partial_{p_{i}} \epsilon_{i}\left(p_{i}\right)-\partial_{p_{j}^{*}} \epsilon_{j}\left(p_{j}\right)\right)^{-1}\right\}
\end{aligned}
$$

or

$$
\left(G_{4}-\partial_{E} F\right)=\partial_{\zeta} F \cdot\{\cdots\} \text { for all } i \neq j, v=1,2,3 \text {. }
$$

If we can show that $\partial_{\xi_{\alpha}} F=0$ for all $\alpha$, we have shown that $F$ does depend only on $E$ and $P$. For technical reasons we will now choose an especially well-adapted local coordinate system, i.e., in addition to $P, E$, we can take, e.g.,

$$
\zeta_{1}:=p_{2}^{2}, \ldots, \xi_{3 n-4}:=p_{n}^{3} \text {. }
$$

The map: $\left(p_{1}, \ldots, p_{n}\right) \rightarrow\left(P_{2}, p_{2}, \ldots, p_{n}\right)$ is a diffeomorphism. The same holds for the map: $\left(P, p_{2}, \ldots, p_{n}\right) \rightarrow\left(E, P, p_{2}^{2}, \ldots, p_{n}^{3}\right)$ [with $E$ understood as the function $\epsilon_{1}\left(P-p_{2}-\ldots-p_{n}\right)$ $\left.+\epsilon_{2}\left(p_{2}\right)+\ldots+\epsilon_{n}\left(p_{n}\right)\right]$ around points where $\partial \epsilon_{1} / \partial p_{2}^{1}$ $\neq 0$. Since there is nothing special about the coordinate $p_{2}^{1}$ it is enough to have $\partial \epsilon_{i} / \partial p_{i} \neq 0$ at some point and for some index $i$ in order that the following holds true in a neighborhood of that point.

In this new coordinate system the curly bracket of (3.14) becomes particularly simple. Making the special choice $p_{i}^{v}=p_{1}^{1}, p_{j}^{v}=p_{2}^{1}$ we have $\left(\partial_{p_{1}^{1}} \xi-\partial_{p_{2}^{1}} \zeta\right) \equiv 0$ and hence

$$
G_{4}=\partial_{E} F \text { on } U \subset M \text { ! }
$$

Inserting this into (3.12) we get

$$
\partial_{P} F+\partial_{\zeta} F \cdot \partial_{p_{i}} \xi=\mathbf{G} \text { for all } i \text {. }
$$

With $i=1$ we get $\partial_{p} \xi=0$ (since $\xi$ depends by definition only on $p_{2}^{2}, \ldots$ ). That is, we have by the same token

$\mathbf{G}=\partial_{P} F$ which implies $\partial_{\xi} F \cdot \partial_{p_{1}} \zeta=0$ on $U$ for all $n$ ?

From this we can infer that $\partial_{p_{i}^{k}} F=0$ for $3(i-1)+v>5$, that is, $F$ does not depend on $\zeta$ at all, in other words,

$$
F\left(p_{1}, \ldots, p_{n}\right)=F(E, P) \text {. }
$$

The proof of the second part of the theorem is almost entirely a consequence of the structure formula (3.19), i.e., given (3.19) the statement is more or less independent of the specific context under discussion. Let $F\left(p_{1}, \ldots, p_{n}\right)$ have the functional form

$$
\left.F\left(p_{1}, \ldots, p_{n}\right)=F(E, P)=\sum_{\left\{i_{1}, \ldots, i_{m}\right\}} f(\ldots\}\right)\left(p_{i_{1}}, \ldots, p_{i_{m}}\right), \quad m<n,
$$

with $F \in C^{m}\left(R^{3 m}\right)$. From (3.20) we infer that

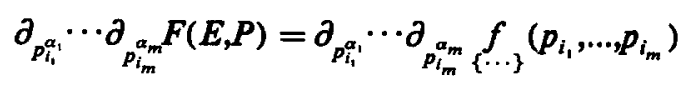

holds for an arbitrary but fixed index set $\left\{i_{1}, a_{1} ; \ldots ; i_{m}, a_{m}\right\}$.

The following calculation can be done without difficulty for an arbitrary $m<n$. But as there is the risk that the degree of notational complexity obscures the basically simple idea underlying the proof, we prefer to give the detailed calculation only for the case $m=2$ and hope to convince the reader that at every step of the reasoning one could replace the number 2 by an arbitrary $m$. For $m=2$ we have

$$
\begin{aligned}
\partial_{p_{i}^{\alpha}} \partial_{p_{j}} F & (E, P) \\
= & \partial_{P^{\alpha}} \partial_{p^{\beta}} F(E, P)+\partial_{E} \partial_{P^{\beta}} F(\cdots) \cdot \partial_{p_{i}^{\alpha}} \epsilon\left(p_{l}\right) \\
& +\partial_{E} \partial_{P^{\alpha}} F(\cdots) \cdot \partial_{p_{j}^{\epsilon}} \epsilon\left(p_{j}\right) \\
& +\partial_{E} \partial_{E} F(\cdots) \cdot \partial_{p_{i}^{\alpha}} \epsilon\left(p_{i}\right) \cdot \partial_{p_{j}^{\beta}} \epsilon\left(p_{j}\right) \\
= & \partial_{p_{i}^{\alpha}} \partial_{p_{j}^{\beta}} f(\cdots\}
\end{aligned}
$$

With 2 (and in general $m$ ) smaller than $n$ we can vary $E$, $P$ while keeping $p_{i}, p_{j}$ fixed! Doing this we see that because the rhs of the second equality has to remain constant

$$
\begin{aligned}
& \partial_{P} \partial_{P} F(E, P)=\text { const, } \\
& \partial_{E} \partial_{P} F(\cdots)=\text { const, } \\
& \partial_{E} \partial_{E} F(\cdots)=\text { const }
\end{aligned}
$$

in other words,

$$
\begin{aligned}
F(E, P)= & A_{\alpha \beta} P^{\alpha} \cdot P^{\beta}+B_{\beta} E \cdot P^{\beta} \\
& +C \cdot E \cdot E+\text { lin. terms + const., }
\end{aligned}
$$

where now $A_{\alpha \beta}, B_{\beta}, C, \ldots$ are constants. For $f_{\{\ldots\}}^{f}\left(p_{i}, p_{j}\right)$ we have

$$
\begin{aligned}
\underset{\{\cdots\}}{f}\left(p_{i}, p_{j}\right)= & A_{\alpha \beta}\left(p_{i}^{\alpha} p_{i}^{\beta}+p_{i}^{\alpha} p_{j}^{\beta}+p_{i}^{\alpha} p_{j}^{\beta}+p_{j}^{\alpha} p_{j}^{\beta}\right) \\
& +B_{\beta}\left(\epsilon_{i} p_{i}^{\beta}+\epsilon_{i} p_{j}^{\beta}+\epsilon_{j} p_{i}^{\beta}+\epsilon_{j} p_{j}^{\beta}\right) \\
& +C\left(\epsilon_{i}^{2}+2 \epsilon_{i} \epsilon_{j}+\epsilon_{j}^{2}\right) \\
& + \text { lin. terms }+ \text { const. }
\end{aligned}
$$

This proves the theorem.

In concluding this section we want to give special emphasis to the following observation which can be extracted 
from the above proof.

Observation: $G_{4}\left|M=\partial_{E} F, \mathbf{G}\right| M=\partial_{P} F$, which shows (cf. Theorem 1 above) that $G_{4}, \mathbf{G}$ are in fact invariants being directly related to $F$ itself !

\section{THE PHYSICAL SIDE OF THE PROBLEM}

We have now to discuss the physical soundness of the crucial assumption being made in connection with scattering invariants of the first kind, namely that the possible scattering events are assumed to cover a full open neighborhood $U$ on $M$. We start again with the simplest case, two particle scattering in momentum space. Assuming $\epsilon(p)=p^{2}$ $(m=1), M \subset \mathbb{R}^{12}$ is given by

$$
p_{1}+p_{2}-p_{1}^{\prime}-p_{2}^{\prime}=0, \quad p_{1}^{2}+p_{2}^{2}-p_{1}^{\prime 2}-p_{2}^{\prime 2}=0,
$$

i.e., $M$ has dimension 8 . The ingoing momenta $p_{1}, p_{2}$ can be freely chosen, which yields six degrees of freedom.

The two missing local coordinates on the manifold $M$ can be found as follows. The asymptotical kinematics is described by $(t \rightarrow-\infty)$

$$
x_{i}^{0}(t)=p_{i} \cdot t+x_{i}^{0}
$$

For a rotational symmetric pair potential scattering in the center of mass system takes place in a fixed plane $\left[\left(x_{1}-x_{2}\right)\right.$ $\cdot\left(x_{1} \times \dot{x}_{1}+x_{2} \times \dot{x}_{2}\right)=\left(x_{1}-x_{2}\right) \cdot L=\left(\dot{x}_{1}+\dot{x}_{2}\right)\left(x_{1} \times x_{2}\right)$; with $\dot{x}_{1}+\dot{x}_{2}=0$ we have $\left(x_{1}-x_{2}\right) \cdot L=0$ ]. The outgoing momenta $p_{1}^{\prime}, p_{2}^{\prime}$ are then uniquely given (at least locally) for given ingoing momenta $p_{1}, p_{2}$ by fixing the relative position at time zero, i.e.,

$$
d:=\left|x_{1}(0)-x_{2}(0)\right| \text { and } \theta:=\left(x_{1}(0)-x_{2}(0)\right) \cdot p_{1}(0),
$$

where $d$ is the distance and $\theta$ is the "angle" between the interacting particles at $t=0$. These two paramenters can be independently varied (at least for non-nasty potentials) via the initial conditions $\left(x_{1}^{0}, x_{2}^{0}\right)$. Then $d$ and $\theta$ can be used as local coordinates on $M$ spanning together with $\left(p_{1}, p_{2}\right)$ a full local coordinate system.

Thus we see that for two particle scattering and physically well-behaved potentials the preassumption of the existence of an open neighborhood $U$ on $M$ where concrete scattering takes place seems to be sound. Similar reasoning can be used for $n>2$ particles in the case of a pure momentum dependence. But note that in any case $x_{\mathrm{in}}^{1}, x_{\mathrm{in}}^{2}$ are related to $x_{1}^{0}, x_{2}^{0}$ via the Möller transformation $\Omega_{+}$, which is in general not simple. That means that the above reasoning, i.e., that by varying $x_{1}^{0}, x_{2}^{0}$ we can appropriately vary $x_{\mathrm{in}}^{1}, x_{\mathrm{in}}^{2}$ and hence $p_{1}^{\prime}, p_{2}^{\prime}$, is physically plausible but not mathematically proved. On the one side one can prove this for a large class of potentials, on the other there may be potentials where this does not hold, e.g., that by varying $x_{1}^{0}, x_{2}^{0}$ we get only a onedimensional set of $\left(p_{1}^{\prime}, p_{2}^{\prime}\right)$ for fixed ingoing momenta $\left(p_{1}, p_{2}\right)$ s.t. $U$ would have a dimension smaller than $M$ itself. This is an interesting question which deserves a separate investigation.

The situation is completely different for more general invariants, depending on momenta and positions. Within the regime of classical nonrelativistic point mechanics there are ten a priori conservation laws, i.e., besides energy momentum, $L=\Sigma_{i} x_{i}(t) \times p_{i}$ and center of mass $S=\Sigma S_{i}(t)$,
$S_{i}(t)=m_{i} x_{i}(t)-p_{i} \cdot t$. For the free motion this reduces to $\Sigma_{i} m_{i} x_{i}^{0}$. There are a couple of subtleties concerning the structure of the manifold $M$ in this general case which will be discussed in the next chapter.

It will turn out that after certain modifications, $M$, defined by the above-mentioned conservation laws, is a $(12 n-10)$-dimensional set in $\mathbb{R}^{6 n} \times \mathbb{R}^{6 n}$. In contrast, however, to the pure momentum case, by fixing the initial conditions of the ingoing particles the initial conditions of the outgoing particles are uniquely given. That is, the manifold of physical interest, $\boldsymbol{M}_{p}$, has only $6 n$ dimensions, i.e., we have the following lemma.

Lemma 2: $M_{p}$, the manifold given by the set of pairs in $\mathbb{R}^{6 n} \times \mathbb{R}^{6 n}:\left\{x^{0} p ; S\left(x^{0} p\right)\right\}, x^{0}=\left(x_{1}^{0}, \ldots, x_{n}^{0}\right)$, etc., $S$ viewed as a map: $\mathbb{R}^{6 n} \rightarrow \mathbb{R}^{6 n}$, is a true submanifold of the manifolds $M$ defined by the conservation laws. The dimensions are $6 n<12 n-10$ for $n \geqslant 2$.

This shows that there is no chance to cover a full open neighborhood $U \subset M$ with real scattering processes for a fixed given interaction potential. Before we discuss the possible consequences of this fact we would like to make an aside about the use of cluster properties in this context.

Definition 6: We say the scattering transformation $S_{n}$ (i.e., for $n$ particles) clusters if the following holds: with $\inf _{j \neq i_{0}}\left|x_{i_{0}}-x_{j}\right| \rightarrow \infty$ for each position $x_{i_{0}}$ we have

$$
\begin{aligned}
& S_{n}\left(x_{1} p_{1}, \ldots, x_{i_{0}} p_{i_{0}}, \ldots, x_{n} p_{n}\right) \\
& \quad \rightarrow S_{n-1}\left(x_{1} p_{1}, \ldots, x_{i_{1}} p_{i_{0}}, \ldots, x_{n} p_{n}\right) \times S_{1}\left(x_{i_{0}} p_{i_{0}}\right),
\end{aligned}
$$

where

$$
S_{1}\left(x_{i_{0}} p_{i_{0}}\right)=\left(x_{i_{0}}^{\prime} p_{i_{0}}^{\prime}\right)=\left(x_{i_{0}} p_{i_{0}}\right) .
$$

That is, one can shift one of the ingoing particles to infinity s.t. in the limit it is not scattered at all. As is well known this cluster property is connected with the range of the interaction. Long range potentials have to be treated by a modified approach.

If we are in a situation where (4.2) holds we can proceed as follows. Restricting ourselves for simplicity for the moment to additive invariants we simply proceed by induction starting from $n=2$. Assuming that for $n=2$ we can, e.g., prove a certain structure of the collision invariants we treat the case $n=3$ by shifting $\left|x_{3}^{0}\right|$ (resp. $\left|x_{1}^{0}\right|, x_{2}^{0} \mid$ ) to $\infty$. Both for the two particle cluster and for the remaining single particle one we can employ the already proved result. We get the corresponding result for $n=3$ by shifting particle (1) [ resp. (2), (3) ] back from infinity to their original positions, thus proceeding from an arbitrary $n-1$ to $n$.

That is, in some cases one can reduce the analysis to the slightly simpler case $n=2$. But even in this case the dimensions of $M, M_{p}$ differ by 2 as long as the interaction potential is kept fixed. We will show in the next chapter that for collision invariants with $F(s, p)-F\left(s^{\prime}, p^{\prime}\right)$ vanishing on $U$ open in $M$ results similar to the previous ones can be proved $\left[s=\left\{s_{i}\right\}=\left\{m_{i} x_{i}^{0}(t)-p_{i} \cdot t\right\}=\left\{m_{i} x_{1}^{0}\right\}\right]$. On the other hand, it can now happen that the manifold $M_{F}$, defined by $F$, hits $M$ transverally s.t.

$M_{F} \cap M$ contains no $U$ open in $\boldsymbol{M}$ but

$$
M_{p} \subset M_{F} \cap M \text { locally. }
$$


This implies in particular that one cannot decide by analyzing the scattering results whether $F$ is an invariant of the first or second kind. As was argued after Definition 4, Sec. II, this may have important consequences. If $M_{F} \cap M$ has a lower dimension than $M$ theremay exist additional hidden symmetries being related to the corresponding transversal local coordinates defining $M_{F} \cap M$, that is, symmetries that show only up on $M_{p}$. Whether $M_{p}$ can in particular support additional additive invariants is then a subtle question which depends on whether the detailed structure of $M_{p}$ and $M_{F}$ allows for an embedding $M_{p} \subset M_{F}$ locally in this special case (due to the assumed additivity $M_{F}$ is relatively "flat," cf. Ref. 14).

Remarks: As to the use of cluster properties of the $S$ matrix in this context we would like to add the following remark. Buchholz kindly informed us that the observation that things become simpler if one exploits the spacelike cluster properties of $S$ was already made by the authors of Ref. 17 a couple of years ago without being published. It is an important tool in their paper. ${ }^{17}$ Unfortunately we were not aware of this fact.

As a last point to mention, in the special case of additive conservation laws, the as yet unpublished results of Ref. 15 may have a certain bearing on the discussion of this chapter. By exploiting what we called above the relative "flatness" of the manifold $M_{F}$ in the case of additive invariants one can possibly prove various results without assuming that certain sets on $M$ are open.

\section{THE CASE OF GENERAL COLLISION INVARIANTS}

Discussion of the case of general invariants of the $S$ transformation, depending on positions, momenta, and the time, needs more care. In a first step we have to define a manifold $M$ adapted to our purpose. Since $\Sigma x_{i}(t) \times p_{i}$ $=\Sigma x_{i}^{0} \times p_{i}, \Sigma m_{i} x_{i}(t)-p_{i} t=\Sigma m_{i} x_{i}^{0}$ for free paths we can write the ten conservation laws in the following way:

$$
\begin{aligned}
& \sum p_{i}-\sum p_{i}^{\prime}=0, \\
& \sum \epsilon_{i}\left(p_{i}\right)-\sum \epsilon_{i}\left(p_{i}^{\prime}\right)=0, \\
& \sum x_{i}(t) \times p_{i}-\sum x_{i}^{\prime}\left(t^{\prime}\right) \times p_{i}^{\prime}=0, \\
& \sum s_{i}(t)-\sum s_{i}^{\prime}\left(t^{\prime}\right)=0,
\end{aligned}
$$

where $t \neq t^{\prime}$ in general and $x_{i}(t)=x_{i}^{0}+p_{i} / m_{i} \cdot t$.

Remark: To be concrete we discuss only the case of nonrelativistic point mechanics. In case of another type of mechanics the conservation of center of mass has to be expressed differently. But no step in our calculations depends actually on the concrete form of the conservation law of center of mass or of the functional dependence $\epsilon(p)$.

It is a simple but important observation that the various time dependencies of the quantities in (5.1) are only a pseudocomplication. In a first step we rewrite the angular momentum conservation law this way:

$$
\sum s_{i}(t) \times p_{i}-\sum s_{i}^{\prime}\left(t^{\prime}\right) \times p_{i}^{\prime}=0,
$$

since $p_{i} \times p_{i}=0$. In a second step we consider the problem in a new space, i.e., the space spanned by the $\left\{s_{i}, s_{i}^{\prime}, p_{i} p_{i}^{\prime}\right\}$. We forget about the time coordinates $t, t^{\prime}$ and observe that (5.1), (5.2) define a $\left(12_{n}-10\right)$-dimensional manifold $M$ in $\mathbf{R}^{6 n} \times \mathbf{R}^{6 n}$, spanned by the coordinates $\left\{s_{i} p_{i} ; s_{i}^{\prime} p_{i}^{\prime}\right\}$.

Definition 7: $M$ is defined by the relations

$$
\begin{aligned}
& \sum p_{i}-\sum p_{i}^{\prime}=0, \\
& \sum \epsilon_{i}\left(p_{i}\right)-\sum \epsilon_{i}\left(p_{i}^{\prime}\right)=0, \\
& \sum s_{i} \times p_{i}-\sum s_{i}^{\prime} \times p_{i}^{\prime}=0, \\
& \sum s_{i}-\sum s_{i}^{\prime}=0
\end{aligned}
$$

in $\mathbf{R}^{6 n} \times \mathbf{R}^{6 n}$, coordinates: $\left\{s_{i}, p_{i} ; s_{i}^{\prime} p_{i}^{\prime}\right\}$.

In a next step we show that collision invariants $F(x(t), p, t)$ are automatically functions of $s, p$.

Lemma 3: A general collision invariant $F(x(t), p, t)$ is a function of $s, p$, that is $F(x(t), p, t) \hat{-} F(s, p)$.

Proof: The proof is simple. We have

$$
\begin{aligned}
F(x(t), p, t) & =F\left(x^{0}, p, 0\right)=F\left(\left\{x_{i}-p_{i} / m_{i} \cdot t\right\}, p\right) \\
& =F(s, p)
\end{aligned}
$$

(identifying the functions for simplicity). We can then prove the following theorem.

Theorem 4: Let $F\left(\left\{x_{i}(t), p_{i}\right\}, t\right) \in C^{1}$ be an invariant of the first kind, i.e., $F(s, p)-F\left(s^{\prime}, p^{\prime}\right) \equiv 0$ on an open neighborhood $U \subset M, M$ defined by Definition 7, then it fulfills the structural relation:

$$
\begin{aligned}
F(s, p) & -F\left(s^{\prime}, p^{\prime}\right) \\
= & G^{v} \cdot\left(\sum p_{i}-\sum p_{i}^{\prime}\right)^{(v)} \\
& +G_{4} \cdot\left(\sum \epsilon_{i}\left(p_{i}\right)-\sum \epsilon_{i}\left(p_{i}^{\prime}\right)\right) \\
& +H_{v} \cdot\left(\sum x_{i}(t) \times p_{i}-\sum x_{i}^{\prime}\left(t^{\prime}\right) \times p_{i}^{\prime}\right)^{(v)} \\
& +K_{v} \cdot\left(\sum s_{i}(t)-\sum s_{i}^{\prime}\left(t^{\prime}\right)\right)^{(v)},
\end{aligned}
$$

with $G^{v}, G_{4}, H_{v}, K_{v}$ in general functions of $s, s^{\prime}, p, p^{\prime}$. Equation (5.4) holds on $\widetilde{U} \supset U, \widetilde{U}$ open in $\mathbf{R}^{6 n} \times \mathbf{R}^{6 n}$.

Proof: Employing Lemma 3 the proof is analogous to the one of Theorem 1. Note that $x_{i}(t) \times p_{i}=s_{i}(t) \times p_{i}=s_{i} \times p_{i}$ for free paths.

If $F$ is only an invariant of the second kind, i.e., $M_{F} \cap M$ has no open neighborhood in $\boldsymbol{M}$ but $\boldsymbol{M}_{F} \cap \boldsymbol{M}_{p}$ contains an open set in $M_{p}$, we would get the following theorem, in general.

Theorem 5: With $M_{F} \cap M_{p}$ containing an open neighborhood $\widehat{U} \subset M_{p}$ we have the corresponding relation on $\widetilde{U} \supset \widehat{U}, \widetilde{U}$ open in $\mathbf{R}^{6 n} \times \mathbf{R}^{6 n}$ :

$$
\begin{aligned}
F(s, p) & -F\left(s^{\prime}, p^{\prime}\right) \\
= & \text { rhs of }(5.5)\}+\sum_{i} \alpha_{i} A_{i}, i=1, \ldots, 12 n-10-6 n,
\end{aligned}
$$

with $\left\{\alpha_{i}\right\}$ functions of $\left\{s, p, s^{\prime}, p^{\prime}\right\}$ and $\left\{A_{i}\right\}$ functions vanish- 
ing identically on $\widehat{U} \subset M_{p}$.

Proof: Most of the proof goes through as above. We only need the following additional lemma.

Lemma 4: Every submanifold of a manifold $M$ is locally cut out by independent functions, i.e., it is the zero set of, e.g., $\left\{A_{i}\right\}$ in addition to the functions defining $M$ itself.

Proof: See, e.g., Ref. 21.

Remark: The possible occurrence of the terms $\alpha_{i} A_{i}$ will, in general, destroy the simple structure given, e.g., in Theorem 3. Note that $A_{i}$ need not even have the form $F_{A_{i}}(p, s)-F_{A_{i}}\left(p^{\prime}, s^{\prime}\right)$.

In the following we will restrict ourselves to invariants of the first kind. We then proceed as in Sec. III. Assuming again that $F$ is twice differentiable we begin with differentiating with respect to $s_{i}$ which turns out to be advantageous. We then restrict again the result to $U \subset M$ s.t. all the terms $\left(\Sigma p_{i}-\Sigma p_{i}^{\prime}\right), \ldots$ vanish indentically. So we get

$$
\left(\partial_{s_{i}} F(p, s)\right)^{(\alpha)} \mid U=\epsilon_{\gamma \alpha \beta} p_{i}^{\beta} \cdot H^{v}+K^{\alpha}
$$

and

$$
\left(\partial_{s_{i}} F-\partial_{s_{j}} F\right)^{(\alpha)} \mid U=\epsilon_{v \alpha \beta} H^{\nu}\left(p_{i}^{\beta}-p_{j}^{\beta}\right),
$$

where $v, \alpha, \beta$, denote the components of the various threevectors and with $\epsilon_{v \alpha \beta}$ the totally antisymmetric three-tensor. Note that $H^{v}$ does not depend on the particular $i, j$ being chosen. Now we differentiate with respect to $p_{i}, p_{j}$, and get

$$
\begin{aligned}
\left(\partial_{p i} F(p, s)\right)^{(\beta)} \mid U= & G^{\beta}+\partial_{p_{i}}^{\beta} \epsilon_{i}\left(p_{i}\right) \cdot G^{4} \\
& +\epsilon_{v \alpha \beta} s_{i}^{\alpha} \cdot H^{v}+K^{\beta}
\end{aligned}
$$

and the analogous expression for the index $\{j\}$. Subtracting the two expressions we get rid of $G^{\beta}$ and can isolate $G^{4}$. As in the pure momentum case we get explicit expressions for all the unknown functions $\left\{G_{4}, G_{\beta}, H_{\beta}, K_{\beta}\right\}$. As in Theorem 1 , Sec. III, we see that all these functions are necessarily scattering invariants on $U \subset M$ !

In the special case of additive invariants we get more detailed information. With $F(p, s)=\Sigma_{i} f_{i}\left(p_{i}, s_{i}\right)$ we get on the lhs of (5.7):

$$
\partial_{s_{i}} f_{i}\left(p_{i}, s_{i}\right)-\partial_{s_{j}} f_{j}\left(p_{j}, s_{j}\right) \text {. }
$$

By the same reasoning as in Theorem 2, Sec. III, we see that the $H_{v}$ are constants [that is, by varying $\left.(i, j)\right]$. Inserting this into (5.6) we see that the $K_{v}$ are constants. We then subtract the expressions (5.8) for the index $i$ (resp. $j$ ) from one another, employing $H_{v}=$ const, $K_{v}=$ const. We get by the same token $G_{4}=$ const, $G_{v}=$ const. That is, we wind up with the following theorem.

Theorem 6: Let $F\left(\left\{p_{i}, s_{i}\right\}\right)$ be a general invariant of the first kind, and $U$ be the corresponding neighborhood on the scattering manifold $M$ defined in Definition 7. Furthermore, we assume $F$ to be twice differentiable.

Then (i) $F$ fulfills Eqs. (5.6)-(5.8) by which the unknown functions $\left\{G_{4}, G_{v}, H_{v}, K_{v}\right\}$ can be determined.

(ii) The $\left\{G_{4}, G^{v}, H_{v}, K_{v}\right\}$ are themselves scattering invariants on $M$, i.e.,

$$
G_{4}\left(p s, p^{\prime} s^{\prime}\right)=G_{4}(p s)=G_{4}\left(p^{\prime} s^{\prime}\right) \text { on } U \subset M .
$$

(iii) In the case of an additive invariant, all these functions are constant.
We get

$$
\begin{aligned}
F(p, s)= & \sum_{i} f_{i}\left(p_{i}, s_{i}\right)=G^{v} \cdot\left(\sum p_{i}\right)^{(v)}+G_{4} \cdot\left(\sum \epsilon_{i}\left(p_{i}\right)\right) \\
& +H_{v} \cdot\left(\sum x_{i}(t) \times p_{i}\right)^{(v)} \\
& +K_{v} \cdot\left(\sum s_{i}(t)\right)^{(v)}+C
\end{aligned}
$$

that is, every additive invariant of the first kind is a superposition of the ten conserved quantities already known.

We want to conclude this chapter with the following theorem which is the analog to Theorem 3 in Sec. III in this general case.

Theorem 7: Let $F(p, s)$, an invariant of the first kind, be twice differentiable, $\partial_{p} \epsilon(p) \neq 0$ a.e., then the following holds.

(i) $F$ is already a function of overall energy, momentum, angular momentum, center of mass, $E, P, L, S$, and we have, in particular,

$$
\partial_{E} F=G^{4}, \quad \partial_{P}^{v} F=G^{v}, \quad \partial_{L}^{v} F=H^{\nu}, \quad \partial_{S}^{v} F=K^{v} .
$$

(ii) If $F$ is an $m$-particle observable with $m<n$ and $F \in C^{m}$, then the $F$ (resp. the corresponding $f$ ) are at most polynomials of $m$ th order in the variables $\left\{P^{\alpha}, E, L^{\beta}, S^{\gamma}\right\}$ (resp. $\left\{p_{i}, \epsilon_{i}, l_{i}, s_{i}\right\}$ ).

Proof: Take Eqs. (5.6)-(5.8). Choose, as in the proof of Theorem III, a new local coordinate system with $E, P, L, S$ representing the first ten coordinates, $\zeta_{11}=p_{2}^{3}, \zeta_{12}=s_{2}^{3}$, etc., view $F$ as a function of these new coordinates, and insert it into (5.6) and (5.8). We get

$$
\begin{aligned}
& \partial_{S}^{\alpha} F+\partial_{L}^{v} F \cdot \epsilon_{v \alpha \beta} p_{i}^{\beta}+\partial_{\zeta} F \cdot \partial_{s_{i}}^{\alpha} \zeta=\epsilon_{v \alpha \beta} p_{i}^{\beta} \cdot H^{v}+K^{\alpha}, \\
& \partial_{P}^{\beta} F+\partial_{E} F \cdot \partial_{p_{i}}^{\beta} \epsilon_{i}\left(p_{i}\right)+\partial_{L}^{v} F \cdot \epsilon_{v \alpha \beta} s_{i}^{\alpha}+K^{\beta} \\
& =G^{\beta}+\partial_{p_{i}}^{\beta} \epsilon_{i}\left(p_{i}\right) \cdot G^{4}+\epsilon_{v \alpha \beta} s_{i}^{\alpha} \cdot H^{v}+K^{\beta} .
\end{aligned}
$$

Choosing, in particular, $s_{i}^{\alpha}=s_{1}^{1}, s_{j}^{\alpha}=s_{2}^{1}, p_{i}^{\beta}=p_{1}^{1}, p_{j}^{\beta}=p_{2}^{1}$, we conclude with the help of expressions (5.7),...,

$$
\partial_{L}^{v} F=H^{v}, \quad \partial_{S}^{v} F=K^{v}, \quad \partial_{P}^{v} F=G^{v}, \quad \partial_{E} F=G^{4},
$$

which shows, as in Theorem III, that $F$ is already a function of $E, P, L, S$ ! The second part of the theorem will be proved exactly along the same lines as in the proof of Theorem III.

Summary and outlook: Since Sec. VI will supply us only with an explicit example of an (even) additive invariant of the second kind for a, however, very special potential, we want to briefly sum up what we have shown above and where future work has to set in.

(i) We have completely classified the scattering invariants depending only on the momenta of the particles in Theorem 3, viz., they are all functions of overall energy and momentum. The same analysis applies to translation covariant invariants in quantum theory and quantum field theory.

(ii) In the case of the more general invariants, depending also on the positions of the particles and the time, we found that they can be grouped into two different classes. For invariants of the first kind we could show again that they are functions of, now, overall energy, momentum, angular 
momentum, and center of mass. The two classes are distinguished by a geometric property, viz., (1) $M_{F}$ contains $M$ locally, or (2) $M_{F}$ intersects $M$ transversally. Since $\operatorname{dim}\left(M_{F}\right)=12 n-1>\operatorname{dim}(M)=12 n-10$, both situations can occur. [In order not to overburden the text we discussed the case (ii) only within the regime of classical mechanics.]

(iii) In case $F$ is an $m$-particle invariant with $m<n$ it could even be shown that $F$ is a polynomial of at most $m$ th order in $E, P, L, S$ in situation (i). We think that the restriction $m<n$ is not superfluous since it might well occur for some special $n_{0}$ that there are invariants depending on all $n_{0}$ particles that cannot be embedded in spaces with $n>n_{0}$ ! (A possible example might be the Runge-Lenz vector.)

(iv) Future work has to deal with these invariants of the second kind mentioned in (ii) (2). In that case the geometry of the intersection of $M_{F}$ and $M$ needs a careful study. The corresponding nonlocal invariants in quantum field theory that are not translation covariant are also studied elsewhere. We would like to mention in this context that it may turn out to be an advantage that our formalism is wide enough to incorporate also charges not commuting with the $S$ matrix. Objects like these may also exist in classical mechanics since invariants of motion (i.e., commuting with $H$ ) do not(!) necessarily commute with the $S$ matrix. In that case more is needed (somewhat sloppily: they should comute as well with $H_{0}$ in the limit $\left.|t| \rightarrow \infty\right)$. Possibly some of the initial conditions of classical mechanics are just of this kind.

\section{AN EXAMPLE OF AN INVARIANT OF THE SECOND KIND}

It is obvious that the notion of an invariant of the second kind is not purely academic. We will see that there exist even additive examples of this type. On the other side; the structure of the manifolds $M_{p}, M, M_{F}$ ( $F$ the invariant) and their mutual intersections are not easy to visualize so that it is quite helpful to get indirect evidence by means of, e.g., Theorem 6.

By Theorem 6 we know that every additive invariant $F$ s.t.

$$
\begin{aligned}
& \left.M_{F} \cap M \supset U \text { (U open in } M\right), \\
& M_{F}:=\text { zero set of } F(p, s)-F\left(p^{\prime}, s^{\prime}\right) \text { in } \mathbb{R}^{6 n} \times \mathbf{R}^{6 n},
\end{aligned}
$$

holds, in other words $M$ being locally contained in $M_{F}$, is necessarily of the form (5.12), that is, with constant coefficients $G, H, K, C$. If for a certain given pair interaction $V$ we can show that there is an additive invariant which is not of the form (5.12) it is necessarily of the second kind, that is,

$M_{F}$ hits $M$ transversally,

$M^{F} \cap M$ contains no open $U$ but

$M_{p} \subset M_{F}$ locally, where $M_{p}:=\{(p, s) ; S(p, s)\}$.
Now take in the two-body case the potential to be $V(r)=C \cdot r^{-2}$. One knows that the so-called time delay $T$ is zero in this case. (For the definition of time delay cf., e.g., Refs. 4 and 6, it can also be found implicitly in Chap. 14 of Ref. 1. See also Appendix B in Ref. 14.) We have

$$
T=\left(s \cdot p-s^{\prime} p^{\prime}\right) / p^{2},
$$

with $s, p, \ldots$, taken in the center of mass system. Calculating $T$ by using the more explicit expression for $T$ with the potential in it (see the above references) we observe that $T=0$ for $V=c \cdot r^{-2}$, i.e., $s_{1} \cdot p_{1} / m_{1}+s_{2} \cdot p_{2} / m_{2}$ is a scattering invariant on $M_{p}$.

On the other side this invariant is not linearly expressible as a superposition of $P, E, S, L$. So it is an explicit example for which the preassumption of the existence of an open neighborhood $U$ on $M$, where $F(s, p)=F\left(s^{\prime}, p^{\prime}\right)$ holds, is not fulfilled.

\section{ACKNOWLEDGMENTS}

Most of this paper was written while I was a guest at the California Institute of Technology. I would like to thank Professor W. A. Luxemburg and Professor B. Simon for their kind hospitality. I would also like to thank the Deutsche Forschungsgemeinschaft for financial support.

${ }^{1}$ L. D. Landau and E. M. Lifschitz, Mechanics (Pergamon, New York, 1960), Vol. 1.

${ }^{2}$ R. Abraham and J. E. Marsden, Foundations of Mechanics (Benjamin, New York, 1967).

${ }^{3}$ W. Thirring, Classical Dynamical Systems (Springer, New York, 1978), Vol. 1.

${ }^{4}$ M. Reed and B. Simon, Scattering Theory (Academic, New York, 1979). ${ }^{5}$ W. Hunziker, in Scattering Theory in Mathematical Physics, edited by J. A. Lavita and J. P. Marchand (Reidel, Dordrecht, 1973).

${ }^{6}$ W. Thirring, in New Developments in Mathematical Physics, edited by $\mathbf{H}$. Miller and L. Pitter (Springer, Berlin, 1981).

'S. Coleman and J. Mandula, Phys. Rev. 159, 1251 (1967).

${ }^{8}$ J. T. Lopuszanski, J. Math. Phys. 12, 2401 (1971).

${ }^{9}$ W. D. Garber and H. Reeh, Commun. Math. Phys. 70, 169 (1979).

${ }^{10}$ J. T. Lopuszanski, "Translation invariant charges in the Q. F. Th. of...," in Proceedings of the International Symposium on Quantum Field Theory, Salerno, Italy, 1985 (North-Holland, Amsterdam, 1986).

${ }^{11}$ E. H. Kennard, Kinetic Theory of Gases (McGraw-Hill, New York, 1939).

${ }^{12}$ A. Sommerfeld, Thermadynamik (Akademie, Leipzig, 1962), 2nd ed.

${ }^{13}$ H. Grad, Commun. Pure Appl. Math 2, 331 (1949).

${ }^{14}$ J. M. Amigo and H. Reeh, J. Math. Phys. 24, 1594 (1983).

${ }^{15} \mathrm{E}$. H. Wichmann, unpublished lecture notes.

${ }^{16} \mathrm{E}$. T. Whittaker, Analytische Mechanik (Springer, Berlin, 1924).

${ }^{17}$ D. Buchholz, J. T. Lopuszanski, and Sz. Rabsztyn, Nucl. Phys. B 263, 155 (1986).

${ }^{18}$ M. Lüscher, Nucl. Phys. B 135, 1 (1978).

${ }^{19}$ D. Buchholz and J. T. Lopuszanski, Lett. Math. Phys. 3, 175 (1979).

${ }^{20} \mathrm{E}$. Hille, Methods in Classical and Functional Analysis (Addison-Wesley, Reading, MA, 1972).

${ }^{21}$ V. Guillemin and A. Pollack, Differential Topology (Prentice-Hall, Englewood Cliffs, NJ, 1974). 